Trinity University

Digital Commons @ Trinity

Psychology Faculty Research

Psychology Department

1982

\title{
Remembering Reactions and Facts: The Influence of Subsequent Information
}

Paula T. Hertel

Trinity University, phertel@trinity.edu

Follow this and additional works at: https://digitalcommons.trinity.edu/psych_faculty

Part of the Psychology Commons

Publication Details

Journal of Experimental Psychology: Learning, Memory, and Cognition

\section{Repository Citation}

Hertel, P.T. (1982). Remembering reactions and facts: The influence of subsequent information. Journal of Experimental Psychology: Learning, Memory, and Cognition, 8(6), 513-529. doi: 10.1037/

0278-7393.8.6.513

This Article is brought to you for free and open access by the Psychology Department at Digital Commons @ Trinity. It has been accepted for inclusion in Psychology Faculty Research by an authorized administrator of Digital Commons@ Trinity. For more information, please contact jcostanz@trinity.edu. 


\title{
Remembering Reactions and Facts: The Influence of Subsequent Information
}

\author{
Paula T. Hertel \\ Trinity University
}

\begin{abstract}
Memory for reactions and judgments about a biographical passage was examined following the presentation of subsequent information relevant to the passage. Experiment 1 demonstrated that reaction memory shifted as a function of the type of subsequent information when 3 weeks separated it from the memory test, but not when testing was immediate or when the information was delivered just prior to the delayed test. These results were obtained again in Experiment 2 and contrasted to shifts in memory for passage facts. Misleading factual information influenced memory for passage facts most when it was delivered just before the delayed recognition test. Similar effects occurred in Experiments 3 and 4 despite changes making the bias and test procedures for reaction and fact memory more comparable. The different ways that memories for reactions and facts are influenced by later information are discussed in terms of the loci of reaction and fact generation (internal and external).
\end{abstract}

When reporting memories of events, we often intersperse our reactions to the event with facts about the event. Within an ongoing narrative we may inform our audience about our feelings or judgments regarding specific aspects of the event, sometimes in an overt fashion ("I was angry that he said that in front of everyone") and sometimes more implicitly ("I knew he should have kept his mouth shut"). Although reactions such as these are sometimes described as products of an affective processing system (Zajonc, 1980), little is known about affective reactions as data within the cognitive system. Even when reactions are defined as idiosyncratic inferences within a strictly cognitive system, we understand little about how they are remembered. Yet, because later events occur to modify our initial reactions, it is reasonable

Experiment 1 was conducted as Experiment 1 of $\mathrm{my}$ dissertation, in partial fulfillment of requirements for the PhD degree from the University of New Mexico. The data from Experiment 1 were reported at the meeting of the Midwestern Psychological Association, St. Louis, May 1980. I am gratful to Linda J. Anooshian, Elizabeth F. Loftus, Richard M. Shiffrin, and Daniel M. Wegner for comments on an earlier version of this article, and to Diane Sausen, Renee Wilson, and Karen Finke for assisting in the collection of data for Experiments 2 and 3. I give a special note of thanks to Henry C. Ellis, whose advice influenced the direction of this research.

Requests for reprints should be sent to Paula T. Hertel, Department of Psychology, Trinity University, 715 Stadium Drive, San Antonio, Texas 78284. to suspect that, in changing our minds, we influence our abilities to accurately recall those initial reactions. Memory for reactions might therefore be susceptible to interference and reconstructive effects like those that operate in memory for factual information.

A variety of experiments have demonstrated that memory for selected aspects of factual information can be influenced or biased by thematically related information encountered later (Dooling \& Christiaansen, 1977; Loftus, Miller, \& Burns, 1978; Schustack \& Anderson, 1979; Snyder \& Uranowitz, 1978; Spiro, 1980). The results of many of these experiments have also suggested that the influence increases with the length of the delay before remembering; an excellent example of this increase in influence over time is provided in Spiro's study of passage memory.

After reading a passage about two college students who fell in love, subjects in Spiro's experiment were incidentally provided with outcome information about the couple's relationship. Outcome information was either consistent with the end of the passage (e.g., the outcome of marriage was consistent with a passage ending that stressed agreement about not having children), or the outcome was contradictory (the outcome of a split in the relationship was contradictory to the passage ending on an agreeable note). Some sub- 
jects had been deceived regarding a later test of passage memory, whereas others believed they were participating in a typical memory experiment. Only the former set of instructions led to errors in passage recall of the type that indicated the influence of the contradictory outcome information, and these errors occurred following either a 3- or 6-week delay, but not after a 2-day delay.

An interesting aspect of Spiro's findings for the present research on memory for reactions concerns instructions to deceived subjects. For these subjects the passage was described as a true story; they were directed to think about the story because they would be asked, ostensibly, to react to it in the second session. Spiro believed that these instructions encouraged the subjects to employ previous cognitive structures and that only under those conditions should evidence for an effect of the outcome be found. What appears equally interesting is that although subjects were never asked to indicate their reactions, their later recall may have reflected those reactions as well as any new reactions resulting from the outcome information. Furthermore, distinguishing between reactions to a passage and facts from the passage may have important implications for understanding the influence of subsequent information on memory.

In the context of passage information, reactions are defined as overt or covert verbal responses that are affectively based and specific to a particular event or type of event described by the passage. First, reactions may contain affective components or express emotions, as do beliefs, judgments, and impressions (Zajonc, 1980). Similarly, reactions may express inferences about the emotions of others, which in turn must be based on stored information of an affective nature. Second, the word reaction implies specificity of the response; the response does not occur "out of the blue" but in reaction to some external event. In this sense, the affective components of reactions may be directly evoked by the event. As a response, a reaction must be generated, constructed or made by the individual. In contrast, facts are not made but exist independently of individual involvement. Facts are objectively verifiable descriptions of events within the passage.
Thus, reactions and facts both may be stored in memory, the first as a representation of how the individual felt or thought (the representation of a construction), the second as the representation of an external state of affairs. (The representation of a fact may be "constructed" or elaborated, but not the fact itself.) Subsequent information may affect memory for the reaction by evoking somewhat different reactions. Subsequent information may affect memory for facts by stating or inferring competing facts. These subsequent reactions or facts may then serve as sources of interference in attempts to remember the original reactions or facts.

In the following experiments, memory for reactions to a biographical passage was investigated by varying the type of outcome information designed to alter reactions. Experiment 1 examined the temporal conditions for influencing memory for reactions. As is the case in memory for facts, longer retention intervals were expected to be associated with larger effects of subsequent information (cf. Spiro, 1980). In addition, the timing of outcome information within the interval was also expected to affect accuracy. Presented immediately prior to the test, episodic memory for the outcome might differentiate reactions to the outcome from previous reactions. Therefore, outcome information was predicted to affect reaction memory to a greater extent when a delay separated the outcome bias and the test. In Experiment 2 , bias effects in memory for reactions were compared with those in memory for facts from the passage. Finally, in Experiments 3 and 4 these comparisons were further explored.

\section{Experiment 1}

A general procedure was developed for measuring reactions and changes in reactions to information presented in a biographical passage. The procedure altered Spiro's methods to include an assessment of reactions (reaction task) following the passage and prior to the presentation of outcome information; the recall task was replaced by a task to assess memory for previous reactions.

Described more completely below, the reaction task required subjects to indicate the 
extent of their agreement with several statements about the characters in the passage. As judged by independent raters, none of the statements referred only to the biographical passage information. Instead, they expressed inferences about the preferences and feeling of the major character. Two forms of the list of statements were available and differed only in the specific wording of the statements. One form served as an indicator of initial reactions; the alternate form was used to test memory for initial reactions. (Forms and purposes were counterbalanced.) In the memory task, subjects were instructed to indicate memory for their initial reactions, rather than reacting anew. Statements in the reaction task were designed to be sensitive to changes in reactions resulting from one of two types of biasing outcomes, presented after the original reaction task. The retention interval and locus of the outcome delivery within the interval were each varied in order to assess previously discussed notions of the time required to accommodate reactions to the outcome.

\section{Method}

Materials. The biographical passage used in all conditions of the experiment (see Appendix) described certain aspects of the life of a hypothetical person, Donna Madison, including her schooling, marriage, husband, children, and hobbies. The passage was constructed to be relatively neutral with respect to each of two outcomes, which in turn corresponded to either a traditional or a nontraditional role for women.

For the reaction task, two lists of 14 statements were constructed. The Appendix provides examples. A scale was provided at the top of each form, indicating judgments of certainly true (1) to certainly false (6). For each form, half of the statements were designed to receive low ratings from a traditional point of view ("She values her role as wife and mother above all"), whereas the other half would deserve low ratings from a nontraditional view ("Donna probably feels that her husband has succeeded at her expense"). The direction of the rating was reversed for each statement on the alternate form. Approximately equal numbers of positive and negative statements were distributed within each half of each form. In general, Form 1 was constructed to avoid extraneous preferences of any kind, and Form 2 contained reversals (affirmation or negation) of statements on Form 1. Statement order was randomized.

Uniform scores for the reaction task were obtained by inverting the ratings for nontraditional statements on each form (1 became 6,2 became 5, . . ., 6 became 1$)$. The inverted ratings were then summed with the noninverted ratings; high scores reflected a nontraditional reaction and low scores a traditional reaction.
Subjects and design. Nine volunteers from introductory psychology classes participated in each of the 12 cells of the factorial design. Factors were bias-test condition (immediate bias and immediate test [II], immediate bias and delayed test [ID], or delayed bias and delayed test [DD]), bias type (traditional or nontraditional), and initial reaction form ( 1 or 2 ). Subjects were required to return for the second session 3 weeks after the first session regardless of test condition, in order to obtain credit in their courses. Those who failed to return were evenly distributed across conditions and were replaced by additional subjects. Approximately equal numbers of each sex participated within conditions.

Procedure. The order and conditions for the procedures in Experiments 1 and 2 are shown in Table 1. At the beginning of the initial session, subjects were told that they were participating in two short studies, the second of which had been delayed for 3 weeks because of a failure to receive all the materials. The first session was described as part of a study about reactions to realworld events as they normally occur outside an experimental setting; for this reason a true story about real people would be used. Subjects were allowed 5 minutes to read and think about the prose passage, and another 10 minutes to complete the reaction forms. This constituted the acquisition phase for all conditions.

Following the collection of reaction forms, the experimenter initiated a pseudo-debriefing session by mentioning that the story had been written about 4 years ago by a graduate student in sociology who was a friend of both the experimenter and the woman in the story. The name of the woman had, of course, been changed. The student had simply wanted to get an idea of how university students would react to various kinds of social information. Recently, she had contacted the experimenter, requesting the collection of more data, because she wanted to find out if various kinds of social changes had occurred on this campus. At this point, subjects in the delayed-bias condition (DD) were dismissed, whereas those in the immediate-bias conditions (II and ID) received biasing information. Subjects selected to hear the traditional bias were told, "In case you'd like to know what happened to the woman in the story, my friend mentioned that she recently had another baby and was really enjoying it. The other kids were getting so big and she had missed having a baby in the house." Subjects slated for the nontraditional bias heard, "In case you'd like to know what has happened to the woman in the story, my friend mentioned that shortly after the story had been written, the woman went back and finished her B.A. She is now a graduate student in physiological psychology in California and she is looking for a research position."

Following delivery of the bias, subjects in the ID condition were dismissed, whereas those in the II condition were tested, with the alternate reaction form, before the session was terminated. Instructions for this condition were as follows:

There is one more task to do today. I have another form, similar to the one you just filled out. I want to make sure that the two forms do not differ. Please try to remember your initial reactions while completing the first form, and base your responses to this form on that memory only. Do not rely on your memory 
for the numbers you chose because this form is worded differently and will not be scored the same. Save your questions until the end, so that you do not influence the way others respond.

During the second session, subjects in the II condition were debriefed, those in the ID condition were tested and debriefed, and those in the DD condition received the bias, were tested, and debriefed. In the DD condition, delivery of the bias occurred following the second sentence of the delayed-test instructions. The bias was modified to indicate that the experimenter had just spoken with the sociology graduate student. These instructions were identical to the immediate-test instructions following a more appropriate introduction:

The materials for the second study never have arrived, but I have come up with a second task for you to do. Do you remember the story you read last time? (Bias for DD condition: Oh, by the way. . . .) Now, I want you to take a minute to remember your reactions to that story. I have another form, similar to the one you filled out last time. I want to make sure that the two forms do not differ. Please try to remember your initial reactions while completing the first form.

Table 1

Order of Procedures in Each Bias-Test Condition for Experiments 1 and 2

\begin{tabular}{|c|c|c|c|}
\hline \multirow[b]{2}{*}{ Session } & \multicolumn{3}{|c|}{ Bias-test condition } \\
\hline & Immediate-immediate & Immediate-delay & Delay-delay \\
\hline \multirow{3}{*}{1} & & Experiment 1 & \\
\hline & $\begin{array}{l}\text { Read and React } \\
(\text { FORM } 1 \text { or FORM 2) }\end{array}$ & $\begin{array}{l}\text { Read and React } \\
\text { (FORM } 1 \text { or FORM 2) }\end{array}$ & $\begin{array}{l}\text { Read and react } \\
\text { (FORM } 1 \text { or FORM 2) }\end{array}$ \\
\hline & $\begin{array}{l}\text { Bias (TRADITIONAL or } \\
\text { NONTRADITIONAL) }\end{array}$ & $\begin{array}{l}\text { Bias (TRADITIONAL or } \\
\text { NONTRADITIONAL) }\end{array}$ & \\
\hline \multirow{3}{*}{2} & Reaction memory test & & \\
\hline & Return only & Reaction memory test & $\begin{array}{l}\text { Bias (TRADITIONAL or } \\
\text { NONTRADITIONAL) }\end{array}$ \\
\hline & & & Reaction memory test \\
\hline
\end{tabular}

Read and react
Extra statements (CONSISTENT/
MISLEADING)
Bias (TRADITIONAL/
NONTRADITIONAL/NONE)

Experiment 2

Reaction memory test

Recognition test

Debriefing

2 Return only

Reaction memory test
Recognition test
Debriefing

Extra statements (CONSISTENT/ MISLEADING)

Bias (TRADITIONAL/ NONTRADITIONAL/NONE)
Read and react

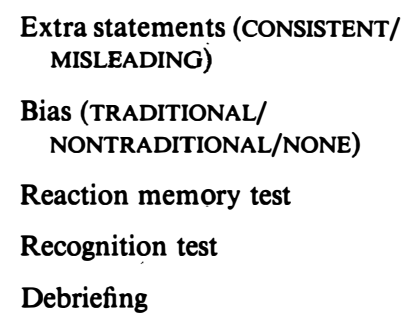

Note. Conditions of all between-groups factors in the design are indicated in uppercase letters. Subjects hearing the consistent extra statements received either the traditional or the nontraditional reaction bias; subjects hearing the misleading extra statements did not receive a reaction bias (NONE). 


\section{Results and Discussion}

A three-way analysis of variance indicated no reliable differences among conditions in initial reactions to the story. In order to evaluate memory effects, difference scores were computed by subtracting the initial reaction score from the test score. Since high reaction scores indicated a nontraditional view, positive difference scores reflected change in the direction of the nontraditional bias. Similarly, negative difference scores reflected change in the direction of the traditional view. ${ }^{1}$

A three-way analysis of variance in difference scores did not reveal a significant main effect of test form. Nor did interactions of test form with the other factors reach significance. Therefore, the data were collapsed across the test-form factor. For the following analyses, values of $p<.05$ were considered reliable, and $M S_{\mathrm{e}}=21.79$.

The results clearly demonstrated an effect of outcome information on memory for previous reactions. Table 2 presents the mean difference scores for each bias type and biastest condition. The effect of the bias was much greater for the ID condition than for the other two bias-test conditions. This result is supported by the reliable interaction of bias type with the planned comparison of ID condition versus II and DD conditions, $F(1$, $96)=6.43$. The overall interaction of bias type with bias-test condition was also reliable, $F(2,96)=3.22$, as was the main effect of bias type, $F(1,96)=19.26$. In order to determine the conditions for which the bias effect occurred, analyses of simple main effects were performed. The bias reliably affected difference scores for the ID condition only, $F(1,96)=21.21$; after 3 weeks the nature of the outcome information was reflected in the subjects' attempts to remember how they had reacted initially. The direction of difference scores in the DD condition was not predicted and perhaps indicated a general forgetting factor. However, many subjects accurately reproduced their original reaction scores.

\section{Experiment 2}

Memory for reactions appears to be vulnerable to interference from related infor-
Table 2

Mean Difference Scores for Each Combination of Bias Type and Bias-Test Condition (Experiment 1)

\begin{tabular}{lrrr}
\hline & \multicolumn{3}{c}{ Bias-test condition } \\
\cline { 2 - 4 } Bias type & \multicolumn{1}{c}{ II } & ID & DD \\
\hline Traditional & -1.39 & -3.78 & -.78 \\
Nontraditional & 1.00 & 3.39 & 1.50 \\
Bias effect & 2.39 & 7.17 & 2.28 \\
\hline
\end{tabular}

Note. Means were computed on the scores of 18 subjects. II = Immediate-immediate; ID = Immediate-delay; $\mathrm{DD}=$ Delay-delay .

mation in ways that may correspond to the effect of intervening materials on memory for facts. Certainly the results of Experiment 1 were consistent with the effects obtained in Spiro's study of passage memory, even though the latter did not include a condition in which outcome information was delivered immediately prior to the delayed recall tests. Delayed-bias conditions, however, were included in two separate investigations of factual memory, one a series of experiments by Loftus et al. (1978) and the other the research of Dooling and Christiaansen (1977).

Loftus et al. presented slides of an autopedestrian accident, followed by a verbal bias either immediately or after various delays associated with the timing of the retention test. The subsequent bias, contained in a questionnaire about the accident, was designed to be misleading or consistent with respect to the original event. If a stop sign was present in the slides, for example, misleading information implied the existence of

\footnotetext{
${ }^{1}$ Note that in the absence of bias effects, difference scores of a nontrivial magnitude should indicate some form of general forgetting. As such, they might be expected to regress toward the mean value of reaction scores and produce a mean difference score around zeró. However, the predicted results should not be confounded by a possible effect of regression toward the mean because initial reactions did not reliably differ across conditions. If subjects hearing a nontraditional bias happened to have produced a more traditional initial reaction mean, for example, a change in the direction of the nontraditional bias would also be considered a change in the direction of the overall initial reaction mean. Mean reaction scores on the memory task were predicted to change in the opposite direction from the mean.
} 
a yield sign. Errors involving false recognition of the yield sign increased with the length of the retention interval, but the largest effect always occurred when the misleading information was presented immediately prior to testing.

A different approach to examining bias effects was taken by Dooling and Christiaansen (1977). Like Loftus et al., they temporally varied the presentation of the bias within the retention interval. However, unlike the results of Loftus et al., their results indicated a larger bias effect when the bias was delivered 1 week prior to testing. Noting this difference, Dooling and Christiaansen suggested that their bias (providing a famous-person name for the previous fictitious passage) may have required "an active cognitive reorganization" of information in memory for the passage. Such a reconstruction is clearly not required by the introduction of the yield sign in the experiments of Loftus et al. Furthermore, presenting a famous-person name (Adolf Hitler or Helen Keller) may have enlisted structures for storing affective reactions and impressions, untapped by misleading inferences concerning traffic signs. The name bias possibly pertained to complex memory structures; the sign bias affected representations for specific aspects of an event. These differences suggest that the mechanisms of influencing memory for reactions might differ from those affecting memory for facts. Yet the modalities for presenting the to-beremembered events and the biases also differed across the studies.

In Experiment 2, misleading information designed to influence memory for facts, or outcome information designed to affect memory for reactions, was orally presented following the same prose passage. All subjects were tested for reaction memory and fact recognition. If different patterns of results were obtained for the two memory measures, the alternative explanation of modality differences would be eliminated, and differing mechanisms for change still suspected.

\section{Method}

Materials. The prose passage and biasing information from Experiment 1 were used in Experiment 2. However, forms for the reaction tasks were shortened to eight statements, preserving the controls for possible extraneous preferences described in Experiment 1. Statements were omitted if they were reported as ambiguous in informal discussions with subjects or if the negation was structurally' awkward. Additional items were eliminated to preserve the traditional/nontraditional balance. Two random orderings of the resulting two forms were used.

As a context for providing the bias regarding memory for passage facts, three additional reaction statements were composed. The first statement (Statement 1 below) did not introduce misleading information or repeat original information; it served as a buffer to avert any suspicion of a bias manipulation. The remaining two statements varied according to condition of fact bias. Statement $2 \mathrm{a}$ was consistent with the passage, which reported Donna's interest in contemporary furniture, whereas statement $2 \mathrm{~b}$ provided misleading information about antiques. Similarly, Statement 3a repeated that Donna had three children (consistent), whereas Statement $3 \mathrm{~b}$ implied that she had only two (misleading). Misleading statements did not directly oppose original information, as did the yield sign in Loftus et al.'s experiments. However, they suggested events in ways that were not entirely compatible with the corresponding details of the passage; for example, we would not consider whether Donna would like to have more than two children, unless we inferred that she had two or fewer. And, at least for those who might agree that she liked to visit antique stores, Statement $2 \mathrm{~b}$ might presuppose an interest in antiques.

1. Donna enjoys spending her time fixing up their house.

2a. Visiting furniture stores is one of her favorite pastimes.

2b. Visiting antique stores is one of her favorite pastimes.

3a. She would like to have more than three children. 3 b. She would like to have more than two children.

The recognition test for passage facts consisted of eight two-alternative forced-choice items. Within each item, the target, a semantically accurate statement of passage information, was paired with an inaccurate distractor; for example, "She was a history major in college" served as a distractor for the statement that her major was English. Neutral items, irrelevant to the reaction statements, occupied the first five positions and the eighth position on the list. They are listed in the Appendix. For Items 6 and 7, distractors referred to the misleading reaction statements described above. These distractors, with target information in parentheses, were the following:

6. Donna and Charles have two (three) children.

7. She has taken decorating courses and knows a lot about antique (contemporary) furniture.

Targets and distractors were presented in an order randomly determined for each pair. A second list of the eight pairs differed from the original in that the withinpair order was reversed.

The final "debriefing" form used in Experiment 2 contained an explanation of the manipulation concerning memory for facts, following a technique employed by Loftus et al. (1978), and instructions to identify which 
information was presented in the passage and which was presented in an additional reaction statement. The debriefing form contained the following information regarding Donna's interest in furniture. (The bias concerning number of children was similarly revealed.)

The study in which you have just been involved was designed to determine the effects of subsequent information on memory. In the beginning you read a story which stated that Donna knew a lot about either antique or contemporary furniture. Later you were asked to react to a statement about shopping at either antique or at furniture stores. Please indicate which information you were given at each point.

I reacted to

I read about a statement about

antique furniture contemporary furniture

antique stores

furniture stores

Subjects and design. Sixteen female volunteers from general psychology classes participated in each of the nine cells of the design. Factors were bias-test condition (II, ID, or DD) and bias type (traditional, nontraditional, or misleading). Subjects hearing either of the reaction biases (traditional or nontraditional) received only consistent information in the additional reaction statements, and thus they served as controls for assessing the bias effects for facts. Subjects who heard the misleading bias for facts received no bias regarding outcome information and served as controls for the reaction bias. Forms for the reaction and recognition tasks were counterbalanced within each cell of the design. Course credit was again made contingent upon second-session attendance; all but two subjects returned and those were replaced.

Procedure. For subjects receiving the traditional or nontraditional reaction bias, all procedures in Experiment 1 were repeated in Experiment 2, augmented by the following. (Refer to Table 1 for a summary.) First, either immediately after the initial reaction task (II and ID conditions) or at the start of the second session (DD condition), the experimenter mentioned that three statements had been mistakenly omitted before duplicating the reaction forms and would now be read. Subjects were instructed to listen to each statement but to write only the number chosen to indicate the extent of agreement or disagreement with each statement. The three consistent statements $(1,2 \mathrm{a}$, and $3 \mathrm{a}$ above) were then read. Responses were written at the bottom of the reaction forms by II and ID subjects and on a blank sheet of paper by DD subjects. Those in the DD condition were asked to take a minute to recall their general reactions before the statements were read, and they were provided with a judgment scale written on the chalkboard. After responses to the additional statements were collected, one of the two reaction biases was delivered.

The remaining addition to the procedures of Experiment 1 consisted of the recognition memory test, which followed the second reaction task in all groups, and was in turn followed by the debriefing task. The instruction for the recognition task was to choose the sentence from each pair which more accurately reflected information they read in the passage. The debriefing form was distributed without comment.
Table 3

Mean Difference Scores for Each Combination of Bias Type and Bias-Test Condition (Experiment 2)

\begin{tabular}{lrrr}
\hline & \multicolumn{3}{c}{ Bias-test condition } \\
\cline { 2 - 4 } \multicolumn{1}{c}{ Bias type } & \multicolumn{1}{c}{ II } & ID & DD \\
\hline Traditional & -.19 & -1.94 & -.13 \\
Nontraditional & .63 & 2.25 & 2.50 \\
Control (misleading & & & \\
$\quad$ fact bias) & 1.06 & .56 & 1.69 \\
$\quad$ Bias effect & .82 & 4.19 & 2.63 \\
\hline
\end{tabular}

Note. The bias effect is determined by subtracting the mean in the traditional condition from the corresponding mean in the nontraditional condition. The effect of misleading information, designed to bias memory for facts but not reactions, can be assessed by comparing the control group means to the midpoints of the traditional and nontraditional means. Means are computed on difference scores from 16 subjects. II = Immediateimmediate; ID = Immediate-delay; DD = Delay-delay.

Treatment of subjects in the three bias-test conditions who received the misleading bias for passage detail differed from the above only in the following ways. First, the additional statements consisted of the buffer statement followed by the two misleading statements $(1,2 b$, and $3 \mathrm{~b}$ above). Second, no outcome information was provided to bias their memory for reactions.

\section{Results}

Memory for reactions. Table 3 provides the mean difference between the reaction memory score and the original reaction score for each cell in the design. Although the bias effect in this experiment was weaker than the corresponding effect in Experiment 1, as demonstrated by the lack of a reliable interaction of bias type with bias-test condition, the same pattern holds: The simple main effect of the bias was reliable only for subjects who heard the bias 3 weeks prior to the delayed memory test, $F(2,135)=4.507, M S_{\mathrm{e}}=$ 18.496. As in Experiment 1, initial reaction scores did not reliably differ among conditions. Nor did misleading information regarding facts lead to reliable changes in memory for reactions across the bias-test conditions.

Recognition of facts. Table 4 presents mean percentage correct recognition for Neutral Items $1-5$ and 8 and Biased Items 6 and 7. All subjects who heard outcome in- 
Table 4

Mean Percentage Correct Recognition of Neutral and Biased Items as a Function of Bias Type and Bias-Test Condition (Experiment 2)

\begin{tabular}{lccc}
\hline & \multicolumn{3}{c}{ Bias-test condition } \\
\cline { 2 - 4 } Items/bias & II & ID & DD \\
\hline Neutral & & & \\
$\quad$ Consistent bias & 95.8 & 78.1 & 78.6 \\
$\quad$ Misleading bias & 96.9 & 78.1 & 79.2 \\
Biased & & & \\
$\quad$ Consistent bias & 87.5 & 65.6 & 64.1 \\
$\quad$ Misleading bias & 84.4 & 37.5 & 18.8 \\
\hline
\end{tabular}

Note. The data of 16 subjects contributed to each misleading-bias mean. Means for consistent-bias groups were combined from the traditional and nontraditional reaction-bias groups and were therefore based on 32 subjects each. Percentage correct recognition was computed on six neutral items and two biased items. II = Immediate-immediate; ID = Immediate-delay; DD = Delaydelay.

formation to bias reactions were given consistent information regarding facts. Since recognition differences between traditional and nontraditional outcome groups were not reliable and did not reliably interact with the other factors, the data from these groups were combined into a consistent-bias category.

An analysis of variance, with between-subjects factors of bias type (consistent or misleading) and bias-test conditions (II, ID, or DD) and the within-subjects factor of item type (neutral or biased), revealed a reliable three-way interaction, $F(2,125)=4.151$, $M S_{\mathrm{e}}=.112$. This interaction can be under- stood to partially reflect a reliable interaction of bias type with a linear trend in bias-test condition for biased items only, $F(1,135)=$ 9.123, $M S_{\mathrm{e}}=.088$. It is clear that the effect of misleading information depended on both the delay of the bias and the delay of the test. In contrast, correct recognition of neutral items was uninfluenced by misleading information and reliably differed according to bias-test condition only, $F(2,135)=22.829$, $M S_{\mathrm{e}}=.022$; percentage correct recognition fell as the retention interval increased.

Judgments on the debriefing form. Table 5 presents the pattern of judgments on the debriefing form, broken down according to whether the subject had correctly or incorrectly recognized the item on the recognition test. The fifth row indicates, for example, that out of the 32 subjects in the delayed consistent-bias condition, 24 correctly recognized Item 6 , and $91.7 \%$ of those 24 were correct again on the debriefing form; $88.2 \%$ of the 17 correct on Item 7 were also correct on the debriefing form. Continuing along the fifth row, all 8 of those who were incorrect on Item 6 and 67.7 of the 15 who were incorrect on Item 7 corrected themselves on the debriefing form; after reading about the nature of the experimental manipulation, these subjects indicated that they had "really" read that Donna had three children or that she was interested in contemporary furniture. In a sense, the debriefing form may have served as a reminder for these subjects. However, when subjects who heard the misleading bias after the delay (the last row of Table 5) were

Table 5

Percentage Correct on Debriefing Form For Recognition Items 6 and 7 (Experiment 2)

\begin{tabular}{|c|c|c|c|c|c|}
\hline \multirow[b]{2}{*}{ Bias-test condition } & \multirow[b]{2}{*}{$n$} & \multicolumn{2}{|c|}{ Correct recognition } & \multicolumn{2}{|c|}{ Incorrect recognition } \\
\hline & & Item 6 & Item 7 & Item 6 & Item 7 \\
\hline \multicolumn{6}{|l|}{ Immediate-immediate } \\
\hline Consistent bias & 32 & $96.6(29)$ & $96.3(27)$ & $66.7(3)$ & $60.0(5)$ \\
\hline Misleading bias & 16 & $100.0(14)$ & $92.3(13)$ & $0 \quad(2)$ & $0 \quad(3)$ \\
\hline \multicolumn{6}{|l|}{ Immediate-delay } \\
\hline Consistent bias & 32 & $100.0(24)$ & $94.4(18)$ & $87.5(8)$ & $64.3(14)$ \\
\hline Misleading bias & 16 & $80.0(5)$ & $100.0(7)$ & $9.1(11)$ & $11.1(9)$ \\
\hline \multicolumn{6}{|l|}{ Delay-delay } \\
\hline Consistent bias & 32 & $91.7(24)$ & $88.2(17)$ & $100.0(8)$ & $67.7(15)$ \\
\hline Misleading bias & 16 & $66.7(3)$ & $100.0(3)$ & $15.4(13)$ & 23.1 (13) \\
\hline
\end{tabular}

Note. The number of correct or incorrect responses on the recognition item is provided within parentheses. In each case, percentage correct on the debriefing form was computed on this number. 
provided with the same reminder and explanation, only $15.4 \%$ of the errors on Item 6 and $23.1 \%$ of errors on Item 7 were corrected (changed on debriefing form). In general, the last two columns of Table 5 show that most recognition errors by subjects hearing the consistent bias were corrected after debriefing but that only 7 of a total of 51 errors in misleading-bias conditions were corrected.

\section{Discussion}

Results concerning memory for reactions essentially provide a replication of Experiment 1 ; the bias effect occurred only for the ID condition. Differences between bias types appeared smaller than in Experiment 1; this perhaps reflects the decrease in the number of items on the reaction sheet or gender differences. (Subject availability dictated that only women participated in Experiment 2.) However, the more important point is that the pattern of results remained the same.

In contrast, a very different pattern of results emerged from the recognition test. Misleading information influenced memory for passage facts after the 3-week delay and to a greater extent when it was provided immediatelybefore the delayed recognition test. These results, obtained from a verbal bias for verbal material, are very similar to the results of Loftus et al. (1978). Both sets of results concern memory for factual details, influenced by implications of inconsistent information. We all know, for example, that stop signs and yield signs do not share the same corners at intersections. Nor do people have two children and three children (unless they have five children). And, to some extent, people preferring contemporary furniture do not search for antiques. When compared with the effects on memory for reactions, these substitutional bias effects appear much more straightforward or direct. Moreover, rather than amounting to response bias effects, the recognition changes appear to occur on a memorial level, as demonstrated by debriefing data in Experiment 2 and in the research of Loftus et al.

In the debriefing task of Experiment 2, very few of the recognition errors made by subjects hearing the misleading statements were corrected after the nature of the ma- nipulation was explained. These subjects were probably no more willfully tenacious in their errors than others (who most often chose the correct information on the second try). Instead their confirmed errors may suggest that correct information was no longer accessible. It is also important to notice that this tendency not to correct the error was at least as strong in the immediate-bias, delayed-test condition, in which only 2 of 20 errors were corrected after debriefing compared with 5 of 26 errors in the delayed-bias, delayed-test condition. The effect of the immediate bias similarly cannot be attributed to confusion.

Finally, obtaining a larger bias effect in the delayed-bias condition cannot be attributed solely to a general decline in passage memory. If such a decline invited use of the recent bias, it would do so differentially for Items 6 and 7. Item 6 has higher overall recognition than Item 7 (see Table 5), yet the effect of misleading information is the same. In addition, a general decline should encourage use of the delayed consistent bias to boost recognition accuracy in the DD condition. Yet accuracy did not improve over the ID condition. Although the latter result is puzzling, it denies that the bias effect in the DD condition resulted only from poor passage memory.

\section{Experiment 3}

In Experiment 2, several aspects of the methodology for investigating memory for reactions differed from the corresponding aspects in studying memory for facts. Before much can be said regarding the possibility of different mechanisms in remembering reactions and facts, some of the extraneous aspects of the tasks must be examined. Without pretending to equate the two sets of procedures, Experiments 3 and 4 were designed to make the procedures somewhat more comparable.

In Experiment 2, instructions to remember original reactions may have alerted subjects to disregard subsequent reactions to a greater extent than instructions to remember the story suggested disregarding subsequent "facts." This possible difference in emphasis might contribute to an alternative explana- 
tion for the different patterns of results within delayed-bias, delayed-test conditions when bias and memory instructions were presented contiguously. Differing degrees of episodic distinctiveness of the bias, established through test instructions, could account for the absence versus the presence of bias effects in the DD conditions. Accordingly, one purpose in conducting Experiment 3 was to vary the emphasis of disregarding subsequent information in remembering passage facts. If subjects who are explicitly directed to ignore possibly faulty references to passage facts still make recognition errors based on misleading statements, then episodic distinctiveness becomes less important as a rival explanation for reaction and fact differences.

The second line of reasoning leading to Experiment 3 involved the respective natures of the memory tests. Specifically, the test of memory for reactions appeared to require greater retrieval efforts than did the two-alternative recognition test for facts. Not only were there six alternative ratings, but the choice with respect to any particular item had to be based partially on memory for a more general reaction because the original item was worded differently. In short, remembering reactions may have been made more complex a task than remembering facts. And since the reaction memory test cannot be converted to a two-alternative, forced-choice recognition task without losing all sensitivity, a cued-recall test of memory for facts was included as part of the procedures of Experiment 3. The cued-recall task is clearly more similar to the reaction memory task in the amount of information that must be retrieved than is the recognition task.

\section{Method}

A total of 128 volunteers from general psychology classes participated in the three-factor design, 16 in each combination of bias timing (immediate or delayed), bias type (consistent or misleading), and instructions (previous memory instructions or explicit instructions to ignore all intervening references to story facts). An additional group of 16 subjects served as a no-bias control, to assess the possible facilitative effect of repeating details in the consistent-bias conditions.

The material and procedures of Experiment 2 were employed and modified in the following ways. Immediate-test conditions were omitted, and all conditions were tested after the 3-week delay. No outcome information was provided to bias memory for reactions, al- though the reaction task was used in the first session as a rationale for the experiment and the reaction memory task was used in the second session to assess memory without the intervening reaction bias. The additional reaction items again provided the context for presenting the consistent or misleading bias for facts; they were read at the end of the first session (immediate bias) or at the beginning of the second' session (delayed bias). Then, following the reaction memory test, a cued-recall test of memory for facts preceded the recognition test.

The cued-recall task was introduced with instructions that either duplicated the recognition instructions in Experiment 2 or stressed the importance of ignoring intervening material, as did memory-for-reaction instructions in Experiment 2. In the first case, subjects were asked to remember details from the story and to provide an answer to each question, guessing if all else failed. In the second case, more explicit instructions stated that subjects should base their answers to the questions on their memories of the passage only and should not be influenced by any references to passage information occurring in the meantime. The recall test consisted of eight questions, corresponding to the content and order of items on the recognition test. Questions 1-5 and 8 are listed in the Appendix. Questions concerning biased facts were the following:

6. How many children do Donna and Charles have?

7. What kind of furniture does Donna know a lot about?

Finally, the recognition test was preceded by repeated instructions appropriate to each condition but was modified to describe the forced-choice procedure. It was followed by the debriefing form.

In summary, subjects in the immediate-bias condition read and reacted to the passage, heard the consistent or misleading extra statements, waited 3 weeks, then returned for a reaction memory test, a recall test, recognition, and debriefing. Subjects in the delayed-bias condition did everything in the above order with one exception. They waited 3 weeks before hearing the extra statements, instead of after. In both conditions of bias timing, instructions for the recall and recognition test varied between subjects (see Table 6).

\section{Results and Discussion}

Recognition. Table 7 presents averaged correct recognition of Items 6 and 7, in each combination of bias timing, bias type, and instructions. A three-way analysis of variance revealed a reliable interaction of timing with type, $F(1,120)=3.800, M S_{\mathrm{e}}=.403,{ }^{2}$ which indicates that the misleading bias had a larger effect when it was presented just prior to the delayed test. This outcome replicated the recognition results in Experiment 2. However,

\footnotetext{
${ }^{2}$ The error term is appropriate for analyses performed on numbers of responses, rather than the percentage measure.
} 
the instructions factor did not reliably interact with either timing or type, or produce a reliable effect alone. In fact, the pattern of means presented in the lower half of Table 7 (instructions to ignore) closely resembles that above (previous instructions). Although failure to reject the hypothesis of no differences is just that, it must be noted that the episodic distinctiveness of the bias remains unsupported as an alternative explanation of the reaction-versus-facts differences. This is the case because instructions more similar to those used in the reaction memory task yielded results typical of the memory-forfacts effect.

Unreported in Table 7, the mean percentage correct recognition in the no-bias control group was 56.3; a comparison with performance in conditions receiving a consistent bias indicates no facilitative effect of repetition in the consistent conditions. The lack of a recognition advantage in the delayed consistent-bias condition may indicate that "contemporary furniture" is a difficult detail to remember and one that gets no boost from the consistent statement: Visiting furniture stores is one of her favorite pastimes.
Table 7

Mean Percentage Correct Recognition of Biased Items (Experiment 3)

\begin{tabular}{lcc}
\hline & \multicolumn{2}{c}{ Bias timing } \\
\cline { 2 - 3 } Instructions/bias & Immediate & Delayed \\
\hline Previous instructions & & \\
$\quad$ Consistent bias & 50.0 & 50.0 \\
$\quad$ Misleading bias & 43.7 & 18.8 \\
Instructions to ignore & & \\
Consistent bias & 56.3 & 53.2 \\
Misleading bias & 40.6 & 18.8 \\
\hline
\end{tabular}

Note. Each mean is based on the data from 16 subjects, and percentages are based on two responses per subject.

Recall. Table 8 presents the mean percentage of correct answers to Questions 6 and 7. Again, a three-way analysis of variance showed a reliable interaction of timing with type, $F(1,120)=9.899, M S_{\mathrm{e}}=.285$ (see Footnote 2). In addition, when the data were scored for number of errors reflecting the misleading bias, this interaction was again obtained, $F(1,120)=8.634, M S_{\mathrm{e}}=.497$; the greater number of recall errors was produced by those subjects who heard misleading information, especially if it was delivered just

Table 6

Order of Procedures in Bias Timing Conditions in Experiments 3 and 4

\begin{tabular}{|c|c|c|}
\hline \multirow[b]{2}{*}{ Session } & \multicolumn{2}{|c|}{ Bias timing } \\
\hline & Immediate & Delay \\
\hline \multicolumn{3}{|c|}{ Experiment 3} \\
\hline 1 & $\begin{array}{l}\text { Read and react } \\
\text { Extra statements (CONSISTENT/MISLEADING) }\end{array}$ & Read and react \\
\hline 2 & $\begin{array}{l}\text { Reaction memory test } \\
\text { Recall test (PREVIOUS/IGNORE) } \\
\text { Recognition test } \\
\text { Debriefing }\end{array}$ & $\begin{array}{l}\text { Extra statements (CONSISTENT/MISLEADING) } \\
\text { Reaction memory test } \\
\text { Recall test (PREVIOUS/IGNORE) } \\
\text { Recognition test } \\
\text { Debriefing }\end{array}$ \\
\hline \multicolumn{3}{|c|}{ Experiment 4} \\
\hline 1 & $\begin{array}{l}\text { Read and react } \\
\text { Bias'(TRADITIONAL or NONTRADITIONAL) }\end{array}$ & Read and react \\
\hline 2 & $\begin{array}{l}\text { Reaction test (MEMORY/NEW REACTIONS) } \\
\text { Recognition test } \\
\text { Debriefing }\end{array}$ & $\begin{array}{l}\text { Bias (TRADITIONAL or NONTRADITIONAL) } \\
\text { Reaction test (MEMORY/NEW REACTIONS/DISGUISED) } \\
\text { Recognition test } \\
\text { Debriefing }\end{array}$ \\
\hline
\end{tabular}

Note. Conditions of all between-groups factors in the design are indicated in uppercase letters. 
Table 8

Mean Percentage Correct (and Percentage of Bias Errors) in Recall of Biased Items (Experiment 3)

\begin{tabular}{llr}
\hline & \multicolumn{2}{c}{ Bias timing } \\
\cline { 2 - 3 } Instructions/bias & Immediate & Delayed \\
\hline Previous instructions & & \\
$\quad$ Consistent bias & $25.0(40.6)$ & $34.4(37.5)$ \\
$\quad$ Misleading bias & $40.6(37.5)$ & $9.5(71.9)$ \\
Instructions to ignore & & \\
$\quad$ Consistent bias & $40.6(28.2)$ & $34.4(28.2)$ \\
Misleading bias & $37.5(34.4)$ & $12.5(68.8)$ \\
\hline
\end{tabular}

Note. Means are computed on 16 subjects per condition, and percentages are based on two responses (to Questions 6 and 7) per subject. Mean percentage of responses scored as errors that reflected the misleading bias is presented parenthetically.

before the test. These effects on the number of correct responses and the number of biased errors did not depend on the instructions factor, which did not reliably interact with either timing or type alone. Nor did the instructions factor produce a reliable main effect. In short, the pattern of recall errors was similar to the pattern of recognition results and thereby does not encourage serious consideration of complexity of the memory task as an alternative explanation of the reaction-versus-facts effects.

Debriefing and memory for reactions. Debriefing data were again examined to determine the number of correct responses on the debriefing form as a function of the correctness of recognition. These results were similar to those obtained in Experiment 2. Moreover, they did not reliably vary according to the condition of instructions. Of the subjects who heard misleading information and allowed it to affect recognition performance (incorrect recognition), none in the immediate-bias condition rectified the error on the debriefing form. Three of the 52 errors in the delayed misleading condition were corrected, two of them under explicit instructions to ignore the bias.

Finally, three-way analyses of variance were performed on the original reaction scores and on the difference scores. No reliable effects were obtained with either measure. The grand mean of the difference scores was .932 , a result indicating that some amount of change in the direction of the traditional bias is perhaps built into the design of the reaction task methodology.

\section{Experiment 4}

In Experiment 3, instructions to remember facts were varied with respect to their explicit reference to biasing information, yet differential effects on recall or recognition were not obtained. In Experiment 4, the opposite approach was taken in attempting to make instructions with regard to the bias comparable for reactions and facts. Previous (Experiment 2) instructions to remember initial reactions clearly oriented subjects to disregard any reactions to outcome information, and these instructions were repeated in the memory instructions condition of Experiment 4. However, in another instructions condition, disguised bias, the biasing outcome information was presented as part of instructions to remember initial reactions. This approach obviously could be used only in the delayed-bias condition, but it appeared to be the only reasonable way both to mimic the deceptive conditions for biasing memory for facts and to specify that the task was to remember reactions, rather than to react anew. In this condition of instructions, subjects were not alerted that biasing information was novel or clearly different from passage information. In a similar fashion, subjects in previous experiments had not been alerted that misleading factual information was different from corresponding passage facts.

Two additional aspects of Experiment 4 concerned the nature of the reaction task and the validity of the memory measure. First, additional subjects in each condition of bias timing and bias type were asked to indicate their current reactions to story information during the second session. These second reactions were designed to serve a comparative function regarding the memory scores. In particular, it was assumed in previous experiments that outcome information would necessarily modify reactions; if this were not true, it would be difficult to understand the regularities in the difference score data. Another problem in interpreting the difference scores would result if, in contrast, new reaction scores would turn out to be much dif- 
ferent from the initial reactions-if, for example, they changed in an opposite direction from that predicted for each bias type.

Finally, the procedure in Experiment 4 included a manipulation check in the form of a final debriefing questionnaire. Subjects were asked to indicate the most recent information they had received about the woman in the story; greater inaccuracy was expected from the disguised-bias condition.

\section{Method}

The nature of the disguised-bias condition of the instructions precluded crossing all three levels of instructions (memory, new reactions, and disguised bias) with the bias timing factor. Disguised-bias instructions were administered in the delayed-bias condition of timing only, but for both traditional and nontraditional types of bias. In the nontraditional condition, for example, disguised-bias instructions to remember initial reactions were as follows:

The first thing we want you to do is to try to remember the story you read in the last session and your reactions to that story. I know it's difficult, but I can't say much to cue your memory. Let me just remind you that at the end of the story the woman went back to school and is now a physiological psychologist.

In the same manner, the traditional condition was told that the woman was thrilled about having another baby. Memory instruction, like those in Experiment 2, emphasized memory for initial reactions with no mention of outcome information. Instructions for the new-reactions condition of the instructions factor were uniformly the following:

The first thing we want you to do is to try to remember the story you read in the last session, and to once again indicate your reactions to that story. Be sure to allow your responses on the reaction form to reflect any changes which may have occurred since your previous reactions.

Twelve volunteers participated in each of the 10 cells of the design (two crossed factors of bias timing and bias type with two levels each, and the instructions factor with two levels crossed with the other factors and one level, disguised bias, nested within the delayed timing condition only). The procedure corresponded to that of Experiment 1 in most respects. Immediate-test conditions were omitted. Also, the test for fact recognition followed the reaction-memory test, to again assess possible effects of outcome information on memory for facts. The recognition test was followed by a debriefing form containing these statements:

1. One aspect of this experiment concerns memory for information about Donna's life. Please indicate the most recent information you were given.

(a) She recently gave birth. (b) She returned to college. (c) She developed an interest in psychology. (d) She developed an interest in decorating her home.
Table 9

Mean Difference Scores (Experiment 4)

\begin{tabular}{llc}
\hline & \multicolumn{2}{c}{ Bias timing } \\
\cline { 2 - 3 }$\quad$ Instructions/bias & Immediate & Delayed \\
\hline Memory instructions & & \\
$\quad$ Traditional bias & -.92 & -.33 \\
$\quad$ Nontraditional bias & 3.25 & 1.33 \\
New Reactions instructions & & \\
$\quad$ Traditional bias & -1.00 & -.83 \\
$\quad$ Nontraditional bias & 3.17 & 2.42 \\
Disguised Bias instructions & & -.25 \\
$\quad$ Traditional bias & - & 1.92 \\
$\quad$ Nontraditional bias & - & \\
\hline
\end{tabular}

Note. Data from 12 subjects contributed to each mean.

2. Please indicate the context in which you encountered the information you checked in Item 1.

(a) The information was presented in the story I read. (b) The information was presented by the experimenter.

A correct response to Debriefing Statement 1 (alternative a or b) should reflect memory for the biasing inf ormation itself, whereas selection of one of the incorrect alternatives should indicate that either the specific outcome described in the bias or the timing of its description (after the passage) is not remembered; responses to Debriefing Statement 2, especially by subjects who correctly identified outcome information, should clarify what they interpreted "most recent" to mean. Correct responses to both debriefing questions might provide some indication of episodic memory for outcomes and were expected to occur most frequently under memory and new-reaction instruction, following a delayed bias. Incorrect responses were expected in the disguised-bias condition, as a check on the deception procedure.

In summary, subjects in the immediate-bias condition read and reacted to the passage, heard either the traditional or the nontraditional outcome, waited 3 weeks, and then were tested for either new reactions or memory for initial reactions. Subjects in the delayed-bias condition read and reacted to the story, waited 3 weeks, returned either to hear one of the outcome biases and to react anew, to remember old reactions following the explicit description of a biasing outcome, or to remember old reactions following a bias disguised as part of the instructions. Finally, all subjects took the recognition test and answered the debriefing questions (see Table 6).

\section{Results and Discussion}

Table 9 reports the mean difference scores in each of the 10 cells of the design. Because of missing cells, these results were analyzed as two separate designs, one to compare newreaction instructions with memory instructions in each combination of bias timing and bias type (the top four rows of Table 9) and the other to examine differences among all 
Table 10

Number of Correct Responses on the Debriefing Form in Each Condition of Bias Timing and Instructions (Experiment 4)

\begin{tabular}{lcc}
\hline \multicolumn{2}{c}{ Bias timing } \\
\cline { 2 - 3 } Instructions & Immediate & Delayed \\
\hline & Statement 1: \\
Identifying & \\
most recent information & \\
Memory & 16 & 22 \\
New reactions & 14 & 19 \\
Disguised bias & - & 12 \\
\hline
\end{tabular}

\section{Statement 2: \\ Identifying source \\ (given correct on Statement 1)}

\begin{tabular}{lrr} 
Memory & 12 & 22 \\
New reactions & 11 & 18 \\
Disguised bias & - & 8 \\
\hline
\end{tabular}

Note. The data were combined across bias-type conditions; 24 correct responses; therefore, were possible in each combination of instruction and bias timing.

three instructional conditions within traditional and nontraditional delayed-bias conditions only (the right column of Table 9).

Memory versus new reactions. A threefactor analysis of variance failed to indicate a reliable interaction of timing and type with the instructions factor, even though the pattern of differences suggests such an interaction. Following memory instructions, a reliable bias effect was obtained under immediate-bias conditions only, $F(1,88)=6.007$, $M S_{\mathrm{e}}=17.341$, a finding that replicates previous results. However, under instructions to react anew, the bias effect was reliable when delivered immediately, $F(1,88)=6.007$, as well as when delivered just prior to the second reactions, $F(1,88)=3.666$. These results indicate that outcome information does change reactions and that the change is in the direction previously assumed for each bias type. $^{3}$

Instructional effects for the delayed bias. A two-factor analysis of variance did not reveal a reliable interaction of bias type with instructions. However, it is apparent in the right column of Table 9 that the bias effect under disguised conditions was not reliably different from the effect under memory conditions; neither was reliable. [Employing the new error term still produced a reliable effect for new reactions at delayed testing, $F(1$, 66) $=4.790, M S_{\mathrm{e}}=13.273$.] The lack of a bias effect in disguised-bias conditions, of course, may be attributed to the weakness of the bias, yet the same must then be said about the "disguised" bias for the facts in Experiments 2 and 3 . Although difficult to interpret, this lack of a reaction-bias effect still differs from the replicated fact-bias effect. In making the procedures more comparable, the arrangements of this study did not affect the divergence of outcomes.

Responses on the debriefing form. Table 10 presents the number of subjects in each condition of bias timing and instruction who correctly identified the bias as the most recent information (top half) and the source of the bias, given correct identification of most recent (bottom half). A Pearson chisquare test of independence indicated that subjects receiving an immediate bias and memory or new-reaction instructions were less likely to correctly identify the most recent information than were those receiving a delayed bias $\left(\chi_{1}^{2}=6.544, p<.025\right)$. This manipulation check supports the notion that episodic information about the bias is not readily accessible after 3 weeks. Even among the 30 subjects in immediate-bias conditions who correctly indicated the most recent information (the bias), 7 reported that it had been presented in the story. This constitutes fairly direct evidence for loss of episodic information regarding the bias, considering that similar errors were not made by delayedbias subjects under memory or new-reaction instructions.

In contrast, delayed disguised-bias subjects were often wrong about the most recent information, responding more similarly to immediate-bias subjects than to those in the other delayed conditions. A chi-square test indicated that the three delayed conditions reliably differed in correctness on Statement 1. $\left(\chi_{1}^{2}=11.297, p<.01\right)$. In addition, 4 of the 12 correct responders who had heard the disguised bias believed it had been presented

\footnotetext{
${ }^{3}$ Although identical effects of an immediate bias were not expected for new-reaction and memory instructions, their similarity is not surprising. After 3 weeks subjects should not be able to differentiate between old and new reactions.
} 
in the story, a result somewhat confirming its disguise. More generally, the large number of errors (12 of 24) in identifying most recent information so soon after its delivery suggests that the ruse was on target.

Recognition. Although no reliable effects were obtained with the overall recognition measure, correct recognition of Item 8 ("Donna is basically discontent/content with her life") was significantly related to scores on the initial reaction task $(r=-.24, p<$ $.01)$. Larger initial reaction scores, indicating less traditional reactions, were associated with falsely remembering that Donna was discontent.

\section{General Discussion}

Memory for reactions or judgments concerning events described in a passage apparently is susceptible to interference effects, as is memory for facts from the passage. However, interference with reaction memory was achieved when 3 weeks separated the bias and test, whereas the largest effect on fact memory was obtained when the bias occurred just prior to this delayed test. When various aspects of instructions and testing for reaction and fact memory were made more comparable in Experiments 3 and 4, the divergent pattern of effects still held.

One possible approach for understanding these differences is to focus on the nature of the memory representations and corresponding process assumptions (cf. Anderson, 1978). The initial processing task presumably results in representations of passage information and in representations of reactions to passage information. This distinction may require specification on a theoretical level, and its nature may be associated with the manner in which the information is generated (cf. Jacoby, 1978; Raye, Johnson, \& Taylor, 1.980; Slamecka \& Graf, 1978). Specifically, the distinction between internally and externally generated memory representations appears to be related to the present differences between memory for reactions and memory for facts. According to Raye et al., greater accuracy regarding internally generated representations can be attributed to the greater likelihood that they include information about the processes that produced them. If this is the case for representations of reac- tions, information concerning additional reactions to outcome information and how they might differ from original reactions might be available for some period of time following the presentation of the outcome. In contrast, externally generated representations, such as those comprising passage memory, are not so likely to include information about operations performed on them, such as the storage of inferences based on misleading statements. This line of reasoning leads to the speculation that we pay attention to our processing efforts when asked to think or construct a response but not when asked to read or listen.

What follows from the generation distinction is that available evidence concerning cognitive operations can be used to make judgments required by test conditions. When we are made to think, we remember how we did it. In the present experiments, such evidence presumably is accessible immediately following reaction biases and is useful in distinguishing old reactions from new reactions. After a delay of 3 weeks, subjects no longer appear to be able to make this distinction, perhaps because memory for specific operations, like other kinds of episodic information, is no longer accessible. Similarly, very little information concerning the changes in externally generated passage memory is accessible under any condition, according to the present line of reasoning. The latter notion is supported by the debriefing data in Experiments 2 and 3. Following a description of the manipulation regarding passage memory, subjects were still unaware of having been affected by the bias. Yet their false recognition of misleading information was greatest under delayed-bias conditions. This pattern of results may demonstrate a recency effect of biasing information that emerges when information regarding cognitive operations is unavailable.

Admittedly, the value of the correspondence between the reaction-versus-facts distinction and the generation distinction in accounting for the present differences is not strongly established. The usefulness of the analogy hinges on differential accessibility of information about cognitive operations, metamemorial information. This, of course, provides a possible direction for future research. 
A related issue focuses on the locus of bias generation. In the present experiments, the functional bias in memory for reactions was assumed to be reactions internally generated by outcome information; externally provided details served to bias fact memory. ${ }^{4}$ Perhaps this compatibility in locus of generation is important in obtaining bias effects, particularly in reaction memory. On the other hand, considering the large body of evidence suggesting that internally generated inferences lead to errors in memory for facts (cf. Bransford, Barclay, \& Franks, 1972), perhaps internally generated "biases" will always exert large effects, provided that information about the source of the bias is not accessible.

Still another line of reasoning leading off from the present research involves the ease with which any source of relevant information can be inserted within or added onto the representation of previous information. The nature of reaction memory may logically require restructuring by biasing information, whereas facts may directly replace or strap onto other facts in memory, as well as restructure or reorganize. Potentially, the restructuring operation could require more time to show a bias effect, and replacement operations could produce immediate results. Again, restructuring operation may produce metamemorial effects as well as bias effects. All these issues, and others, remain to be addressed.

A final consideration in the present conceptualization is required for the obvious yet important observation that reactions and facts often differ in memorability. In most of the cases, remembering reactions should be an easier and more successful task than remembering facts. To be more precise, to the extent that reactions and judgments require more cognitive effort (Eysenck \& Eysenck, 1979; Tyler, Hertel, McCallum, \& Ellis, 1979), are associated with a high degree of internal generation (Raye et al., 1980), or involve self reference (Rogers, Kuiper, \& Kirker, 1977), they will be remembered better than facts. This comparison, although difficult to make in specific real-world settings, is quite relevant to the observation that we report our reactions along with the facts about events. It may be that we fill in hazy areas in our memories for facts with easily retrieved memories for reactions to the set of facts as a whole. Furthermore, this line of reasoning also suggests that a bias for reaction memory strengthens that memory, in its altered form, through the very process of restructuring or regenerating. In contrast, a bias in memory for facts typically does nothing but impose a last-minute change.

\begin{abstract}
${ }^{4}$ The source of generating bias information is best conceived on a continuum from internal to external. In Experiments 2 and 3, misleading statements lead to inferences that are more properly identified as the biases than are the statements themselves. However, competing elements in the biases (two children, antiques) are explicitly stated, in contrast to the unidentified elements of the reaction biases, which are entirely generated. A similar continuum underlies the distinction between representations of reactions and facts.
\end{abstract}

\section{References}

Anderson, J. R. Arguments concerning representations for mental imagery. Psychological Review, 1978, 85, 249-277.

Bransford, J. D., Barclay, J. R., \& Franks, J. J. Sentence memory: A constructive versus interpretive approach. Cognitive Psychology, 1972, 3, 193-209.

Dooling, D. J., \& Christiaansen, R. E: Episodic and semantic aspects of memory for prose. Journal of $E x$ perimental Psychology: Human Learning and Memory, 1977, 3, 428-436.

Eysenck, M. W., \& Eysenok, M. C. : Processing depth, elaboration of encoding, memory stores, and expended processing capacity. Journal of Experimental Psychology: Human Learning and Memory, 1979, 5, 472-484.

Jacoby, L. L. On interpreting the effects of repetition: Solving a problem versus remembering a solution. Journal of Verbal Learning and Verbal Behavior, 1978, 17, 649-667.

Loftus, E. F., Miller, D. G., \& Burns, H. J. Semantic integration of verbal information into a visual memory. Journal of Experimental Psychology: Human Learning and Memory, 1978, 4, 19-31.

Raye, C. L., Johnson, M. K., \& Taylor, T. H. Is there something special about memory for internally generated information? Memory \& Cognition, 1980, 8, 141-148.

Rogers, T. B., Kuiper, N. A., \& Kirker, W. S. Self-reference and the encoding of personal information. Journal of Personality and Social Psychology, 1977, 35, 677-688.

Schustack, M. W., \& Anderson, J. R. Effects of analogy to prior knowledge on memory for new information. Journal of Verbal Learning and Verbal Behavior, 1979, 18, 565-583.

Slamecka, N. J., \& Graf, P. The generation effect: Delineation of a phenomenon. Journal of Experimental Psychology: Human Learning and Memory, 1978, 4, 592-604.

Snyder, M., \& Uranowitz, S. W. Reconstructing the past: 
Some cognitive consequences of person perception. Journal of Personality and Social Psychology, 1978, 36, 941-950.

Spiro, R. J. Accommodative reconstruction in prose recall. Journal of Verbal Learning and Verbal Behavior, 1980, 19, 84-95.
Tyler, S. W., Hertel, P. T., McCallum, M. C., \& Ellis, H. C. Cognitive effort and memory. Journal of Experimental Psychology: Human Learning and Memory, 1979, 5, 607-617.

Zajonc, R. B. Feeling and thinking. American Psychologist, 1980, 35, 151-175.

\section{Appendix}

\section{Materials}

\section{Passage}

Donna Madison is thirty years old and has lived in Albuquerque for most of her life. For the last ten years she has been married to Charles Madison, who has worked his way into the upper echelon of a small electronics firm. Donna met Charles in college, where she was an English major interested in twentieth-century American poetry. Charles was an average student but he had strong goals for his career. Donna was a very bright student but seemed to lack a direction in her studies. However, she was certain that she was in love and, since she was raised traditionally, she decided to get married and have a family.

Charles was a graduate student at the time. After their wedding Donna quit school and got a job as a secretary in the Psychology Department, in order to help support them. She worked until Charles finished his degree and she gradually developed an interest in psychology. She thought it would help her understand herself and others better.

Donna quit the job two months before her first baby was born and has spent several years taking care of her family. She now has two boys and a girl, all under the age of seven. The five of them live in a charming old house which they have remodeled on weekends. Donna has taken interior decorating courses and knows a lot about contemporary furniture. Charles is very proud of his wife because she is a very good mother, an excellent hostess, and she provides a calm and well-organized home. Donna is basically content with her life.

\section{Examples of reaction statements}

1. Form 1: Donna doesn't feel that her husband has succeeded at her expense. (negative/agreement from traditional view)

Form 2: Donna probably feels that her husband has succeeded at her expense. (positive/agreement from nontraditional view)
2. Form 1: Being a wife and mother is not the most important thing in her life. (neg. ative/agreement from nontraditional view)

Form 2: She values her role as wife and mother above all. (positive/agreement from traditional view)

3. Form 1: Donna is glad she is able to be home with the children while they are young. (positive/agreement from traditional view)

Form 2: Staying home with preschool children is not one of her priorities. (negative/ agreement from nontraditional view)

\section{Neutral recognition items (Experiments 2-4)}

1. Donna has been married to Charles for (ten/ fifteen) years.

2. She was (an English/a History) major in college.

3. Charles was (a very bright/an average) student.

4. Donna quit work (two/six) months before her first baby was born.

5. She thought psychology would help her understand (her husband/herself and others) better.

8. Donna is basically (discontent/content) with her life.

\section{Neutral questions (Experiment 3)}

1. How many years was Donna married to Charles?

2. What was Donna's major in college?

3. What type of student was Charles?

4. How many months before her first baby was born did Donna quit work?

5. Who did Donna feel psychology would help her understand?

8. How did Donna feel about her life? 